\title{
Juvenile Dermatomyositis and the Inflammatory Myopathies
}

\author{
Collin Swafford, DO ${ }^{1}$ E. Steve Roach, MD ${ }^{1}$ \\ ${ }^{1}$ Department of Neurology, Dell Medical School, University of Texas, \\ Austin, Texas \\ Semin Neurol 2020;40:342-348. \\ Address for correspondence Collin Swafford, DO, Department of \\ Neurology, Dell Medical School, University of Texas, 1701 Trinity St, \\ Stop Z0700, Austin, TX 78712 \\ (e-mail: Collin.Swafford@austin.utexas.edu).
}

\author{
Abstract \\ Keywords \\ - Inflammatory \\ myopathies \\ - juvenile \\ dermatomyositis \\ - inclusion body \\ myositis \\ - immune-mediated \\ necrotizing myopathy
}

The inflammatory myopathies comprise disorders of immune-mediated muscle injury. The histopathology and clinical features help distinguish them. Juvenile dermatomyositis (JDM) is the most common form of myositis in children and adolescents. Children with JDM present with proximal muscle weakness and characteristic rashes. The presentation is similar in children and adults, but JDM is a primary disorder and the adult form often is concerning for a paraneoplastic syndrome. Proximal muscle weakness occurs with dermatomyositis, polymyositis, and immune-mediated necrotizing myopathy, but the latter two conditions have no dermatologic findings or distinct tissue changes which set them apart from dermatomyositis. Inclusion body myositis, also included in the inflammatory myopathies, presents with more distal involvement, and microscopically exhibits identifiable rimmed vacuoles. We review key features of these disorders, focusing in more detail on JDM because it is more often encountered by the child neurologist.
The inflammatory myopathies are characterized by immunemediated skeletal muscle injury. The pattern of muscle involvement along with the histopathological findings helps distinguish the different disorders. Dermatomyositis (DM), polymyositis (PM), inclusion body myositis (IBM), and immune-mediated necrotizing myopathy (IMNM) are predominantly seen in the adult population. Juvenile dermatomyositis (JDM) presents in early adolescence and should be considered when a young patient presents with complaints of muscle weakness and dermatologic changes. We will focus primarily on JDM because it is the inflammatory myopathy most likely to be encountered by the child neurologist. The other inflammatory myopathies will be reviewed briefly, recognizing they are far rarer or do not occur in the pediatric population.

\section{Juvenile Dermatomyositis}

JDM is rare, but it is the most common inflammatory myopathy of childhood. ${ }^{1}$ The incidence of JDM is around 2.5 per million per year, and the prevalence is 2.5 per 100,000 . Median age of onset is between 5.7 and 6.9 years, with median age at the time of diagnosis around 7.5 years. ${ }^{1,2}$ The exact cause of JDM is unknown, but several theories have emerged over the years. Studies point toward a genetic predilection to the disease with specific genetic markers such as HLA-D3, for example. ${ }^{3}$ The predisposition for JDM may be flared by environmental factors such as sun exposure, medications, or certain infections. ${ }^{4}$ Suspected organisms inciting JDM are group A beta hemolytic streptococci, ${ }^{5}$ enterovirus, ${ }^{2}$ and Coxsackie B virus. ${ }^{6}$ The third may serve a clue as to why there seems to be a seasonal nature to the disease with a higher incidence occurring in nonwinter months. ${ }^{7}$ Whether genetically destined or provoked by an environmental or infectious process, an immune-mediated inflammatory cascade is triggered, leading to a systemic vasculopathy affecting the endothelial cells in the majority of a patient's tissues..$^{8}$ Early in the disease course, cytokines and interferons cause a small vessel vasculitis. ${ }^{9}$ Over time, however, inflammatory products occlude the vessels, leading to capillary destruction and surrounding tissue necrosis. ${ }^{9,10}$

Tissue inflammation occurs throughout the body, but is most notable in the skeletal muscle and skin, giving the hallmark clinical features of proximal weakness and characteristic
Issue Theme Pediatric Neurology, Part 2; Guest Editors, Kendall B. Nash, MD, and Nilika Shah Singhal, MD
Copyright $\odot 2020$ by Thieme Medical Publishers, Inc., 333 Seventh Avenue, New York, NY 10001, USA. Tel: +1(212) 760-0888.
DOI https://doi.org/ 10.1055/s-0040-1705120. ISSN 0271-8235. 
rashes. ${ }^{1,2}$ The large muscles of the hip and shoulder are affected in a symmetric pattern, with typically preserved strength in the distal smaller muscles of the hands and feet. ${ }^{1,11}$ In comparison to patients with adult-onset DM and PM, JDM demonstrates the greatest weakness in hip flexors, extensors, and adductors, as well as neck flexors and shoulder abductors. ${ }^{12,13}$ Functional limitations include difficulty in walking, using stairs, or lifting arms above the head. ${ }^{12,14}$ Muscle weakness may progress to the bulbar muscles, leading to dysphonia and dysphagia, and may be heralded by a nasal quality to the voice or coughing with swallowing. ${ }^{15,16}$ Weakness may be an acute presentation or can take several weeks to months to develop, depending on the degree of inflammation. ${ }^{11}$ With progressive disease, the inflammatory changes can lead to fibrosis and flexion contractures of involved muscles, causing further limitations in function. ${ }^{13,17}$

The characteristic dermatologic features of JDM involve the heliotrope rash, Gottron's papules, and nail fold changes. These findings may precede the muscle involvement by several months. The heliotrope rash is described as a purple violaceous rash involving the eyelid and surrounding the orbit. ${ }^{18,19}$ There may be periorbital edema associated with the rash, depending on the severity of disease. ${ }^{20,21}$ The heliotrope rash is not typically seen in other autoimmune disorders such as systemic lupus erythematosus and scleroderma; thus, it is almost pathognomonic for JDM. ${ }^{22}$ Gottron's papules are an erythematous papulosquamous rash of the dorsal aspects of the elbows and metacarpal and interphalangeal joints. ${ }^{2}$ Telangiectasias are commonly seen within Gottron's papules. ${ }^{22}$ Periungual telangiectasias are tortuous capillary vessels noted at the nail folds. Best seen with a capillaroscopy, one can visualize the dilated capillary loops with areas of drop out, or avascularity, directly the cause of endovascular inflammation. ${ }^{23,24}$ Other cutaneous findings include a photosensitive malar distribution rash, poikilodermatous eruption on the chest (V-sign) and back (shawl sign), and alopecia. ${ }^{22}$ These findings are not specific for JDM and require further evaluation to rule out other systemic autoimmune disorders.

Other systems involved in JDM include soft tissues, gastrointestinal tract, and the lungs. Dystrophic calcinosis, or the deposition of calcium products in injured tissue, occurs in up to $40 \%$ of patients with JDM. ${ }^{25}$ Commonly affected areas are skin, subcutaneous tissue, and muscle of the elbows, knees, trunk, hands, and feet. ${ }^{1,9,25}$ Calcinosis is a chronic finding in JDM, typically presenting greater than 1 year from the start of inflammation. ${ }^{25}$ An uncommon but serious finding is vasculopathy in the gastrointestinal tract leading to ulceration, hemorrhage, or perforation. ${ }^{26}$ Interstitial lung disease, seen in only 2 to $14 \%$ of cases, is a rare complication of JDM and may be present with or without overt respiratory symptoms. ${ }^{27,28}$ Lastly, patients with JDM may complain of systemic symptoms such as nonspecific fatigue, adenopathy, fever, or arthralgias. ${ }^{2}$

A thorough history and physical exam will help point toward the diagnosis, as many of the clinical features of the disease are readily noted by gross observation. When the clinical suspicion for JDM is high, laboratory testing and imaging support the clinical diagnosis. Basic screening labs with complete blood count, alanine aminotransferase (ALT), aspartate aminotransferase (AST), creatine kinase (CK), lactate dehydrogenase (LDH), C-reactive protein (CRP), and erythrocyte sedimentation rate (ESR) should be drawn to evaluate for signs of systemic inflammation and chronic disease..$^{29,30}$ The patient may present with anemia of chronic disease; the white blood cell count, CRP, and ESR may be elevated as signs of acute or chronic inflammation. LDH, ALT, and AST may be elevated due to muscle breakdown. ${ }^{29} \mathrm{CK}$ levels can be normal, but in over half of patients with JDM the CK is more than 10 times the upper limit of normal. ${ }^{29,31}$ Myositis autoantibodies (MSAs) may be positive in half of cases, with specific MSAs being anti-Jo-1, anti-p155/140 most frequently, followed by anti-MJ, antisynthetase, antisignal recognition particle (SRP), and anti-MI-2.32 Many individuals, however, do not have a positive autoantibody. Anti-Jo-1, which is commonly positive in adult forms of DM, is rarely positive in pediatric cases. ${ }^{33}$ ANA may be positive as well, but is nonspecific for autoimmune disease.

Radiographic evidence of inflammation may be seen in affected muscles, best seen on MRI. The classic changes seen on MRI are muscle edema, perifascicular edema, or signal changes, and honeycombed appearance of affected muscles (-Fig. 1). ${ }^{34,35}$ The original 1975 Peter and Bohan criteria for the diagnosis of DM included muscle biopsy and electromyography (EMG) in addition to the clinically noted proximal muscle weakness, skin changes, and elevated muscle enzymes. ${ }^{2}$ These are not routinely performed, as the updated criteria by the European League Against Rheumatism and American College of Rheumatology (ELAR/ACR) use clinical and laboratory testing predominantly with biopsy if the diagnosis remains equivocal. ${ }^{36}$ If a biopsy is needed, a muscle that is mild to moderately affected may help solidify the diagnosis in the proper context with supporting data. A profoundly weak muscle may only demonstrate fibrosis and chronic inflammatory changes without the architecture noted in JDM. The typical muscle biopsy (-Fig. 2) demonstrates a perivascular mononuclear cell infiltrate, myofiber necrosis, and atrophy of the perifascicular myocytes. $^{37}$ The infiltrates largely consist of B cells, CD4+ helper T cells, and macrophages. ${ }^{38}$ Furthermore, an EMG and nerve conduction study of a patient with JDM demonstrates small, myopathic motor units with spontaneous discharges, ${ }^{37}$ which supports the pathology being in the muscle itself and not the nerve that supplies it.

The clinical course of JDM presents in one of three paths. A monophasic course, roughly one-third of cases, presents as an initial flare, and remission is achieved in 2 years with or without therapy. A chronic continuous course, occurring in up to two-thirds of cases, demonstrates disease burden despite adequate therapy for greater than 2 years. Last, and considerably less common, is a polyphasic, relapsingremitting course when not treated with corticosteroids. ${ }^{39,40}$ Inadequate therapy or delay in treatment are important factors in dictating the clinical course, while early therapy has shown to decrease morbidity and mortality. ${ }^{41,42}$

Corticosteroids are the initial and mainstay therapy in patients with JDM. ${ }^{2,11,42,43}$ Whether to use oral 


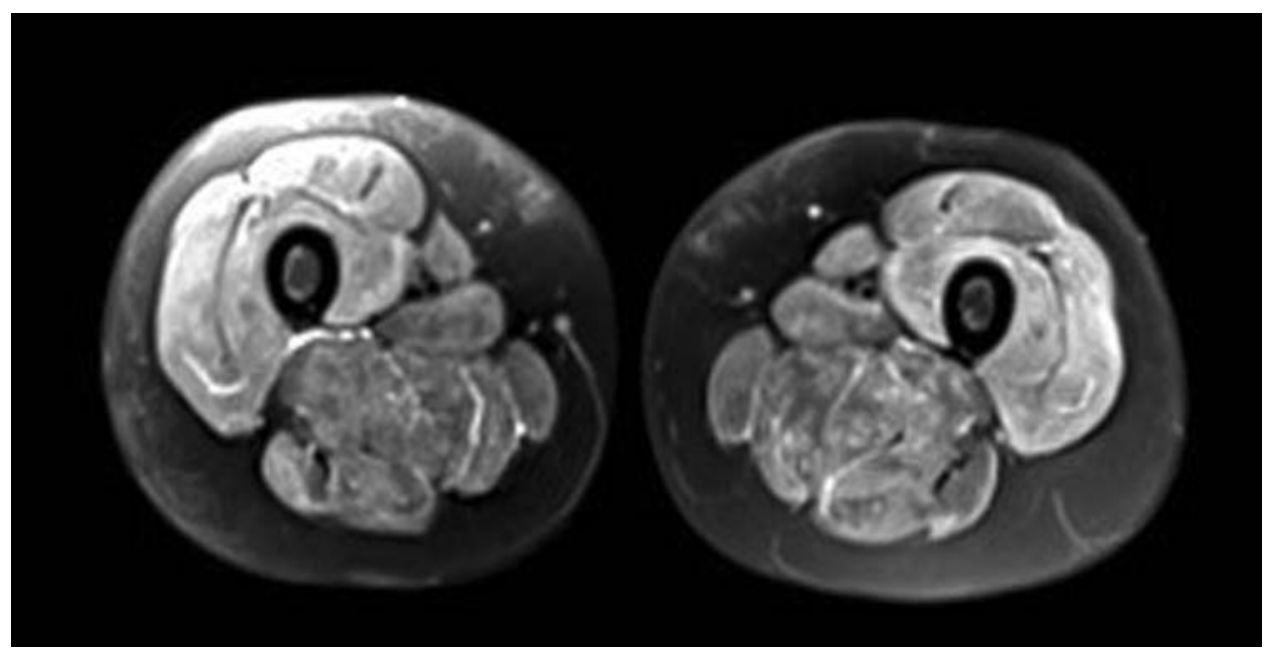

Fig. 1 T2-weighted MRI of the femur of an adolescent with juvenile dermatomyositis. Note the heterogenous signal change in the posterior compartment of the thigh bilaterally.
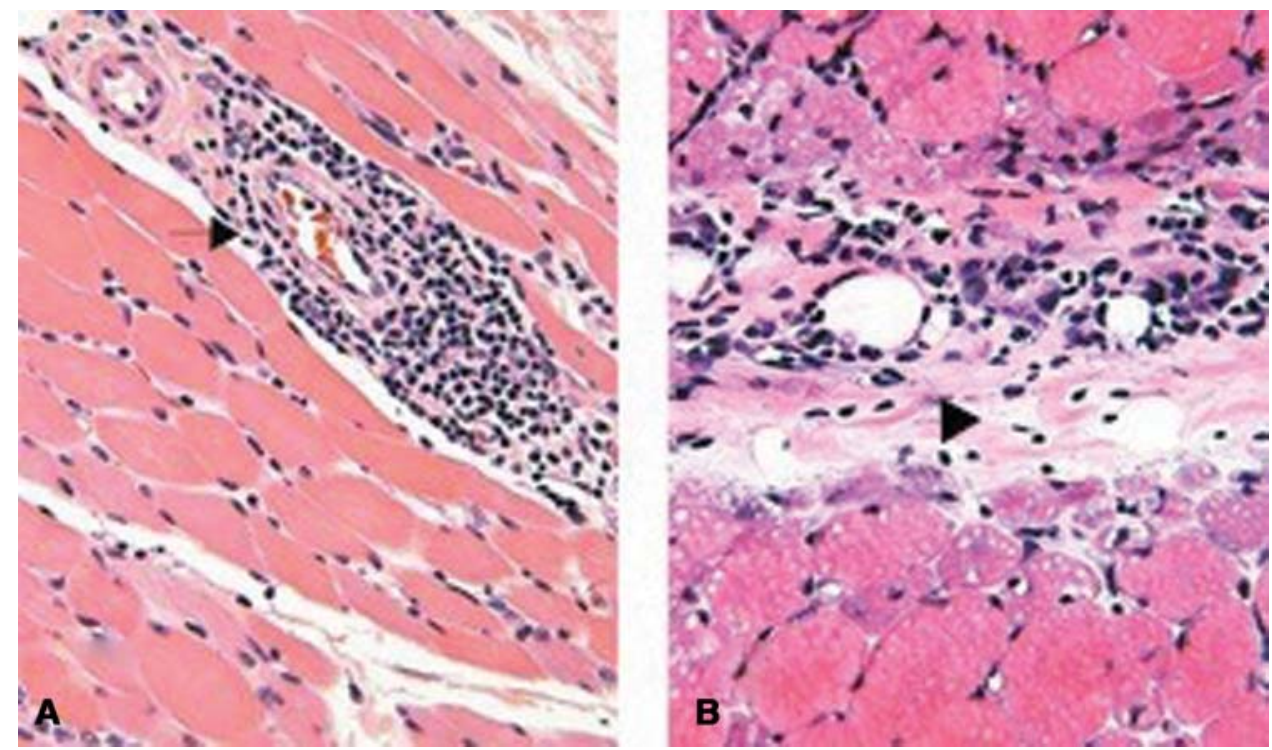

Fig. 2 Muscle biopsy of vastus lateralis of an adolescent with juvenile dermatomyositis. (A) Perivascular inflammatory infiltrates (arrow); (B) perifascicular infiltrates with atrophy of the perifascicular myocytes and relatively preserved architecture of the more centrally located myocytes (arrowhead). (Figures provided by Marc Cohen, MD, Pathologist University Hospitals Cleveland Medical Center.)

prednisolone or intravenous methylprednisolone at the initial presentation depends on the severity of presentation and is institution dependent. A retrospective evaluation of the effectiveness of oral versus intravenous steroids demonstrated a reduction in disease activity and chronic burden with intravenous steroids, specifically for flares. ${ }^{41,43}$ Patients on steroid therapy demonstrate improved energy, increased strength, and decreasing muscle enzymes, which can be followed over time to monitor effectiveness of treatment. ${ }^{37}$ With long-term steroid use, however, patients demonstrate side effects, including weight gain, hypertension, osteoporosis, and steroid myopathy. ${ }^{37}$ The addition of a steroid-sparing agent, such as methotrexate, significantly reduces the overall amount of steroids the patient receives, thus decreasing the effects of chronic steroid use. ${ }^{43}$

Refractory cases of JDM require additional immunomodulating agents to prevent continued deterioration from the disease.
Cyclosporine is an effective alternative to methotrexate as a steroid-sparing agent for daily treatment. ${ }^{44,45}$ Intravenous immunoglobulin (IVIG) has proven efficacious in adults and children with DM. Patients treated with IVIG demonstrated similar disease control or lower disease activity, specifically in those children who were steroid resistant. ${ }^{46}$ In addition to immunosuppression, immunomodulation medications are used in severe refractory cases as well. Rituximab, a B-cell-depleting medication, showed varied results in a randomized control trial, but did demonstrate ability to decrease steroid burden in patients with JDM. ${ }^{47}$ Tumor necrosis factor-alpha (TNF- $\alpha$ ) has been shown to lead to a more chronic course with worsening calcinosis. ${ }^{43,48}$ Infliximab and etanercept, anti-TNF- $\alpha$ agents, have proven effective in the treatment of severe refractory JDM, specifically in joint movement, skin findings, and reduction of calcinosis. $^{39,48}$ Medications used in the treatment of SLE have also shown reductions in disease burden, such as 
mycophenolate mofetil ${ }^{49}$ and tacrolimus, ${ }^{50,51}$ both demonstrating improved skin findings and decreased muscle inflammation.

\section{Dermatomyositis and Polymyositis}

Adult-onset DM behaves in a similar fashion as the juvenile form. The hallmarks of disease are the recognizable heliotrope rash and Gottron's papules, as well as symmetric and proximal muscle weakness. ${ }^{52}$ The findings on muscle MRI, biopsy, and EMG demonstrate the same honeycombed pattern, perifascicular atrophy, and inflammation and myopathic changes, respectively. ${ }^{13,52}$ Similar myositis-specific antibodies are present in children and adults. ${ }^{52,53}$ The complications of the disease, however, are markedly disparate between the two populations, specifically in regard to lung disease and association with malignancy. ${ }^{53}$ Interstitial lung disease is associated with higher mortality in adult patients, and is seen in 20 to $65 \%$ of patients with idiopathic inflammatory myopathies. Although half of children may demonstrate changes on pulmonary function testing, only about $3.5 \%$ of affected children exhibit radiographic evidence of interstitial lung disease. ${ }^{53,54} \mathrm{DM}$ in adults is more likely to be associated with malignancy, with the most common being breast cancer and adenocarcinoma of the lung and colon. The risk is highest in the first year after onset of symptoms. $^{53,55,56}$ The association of improvement of DM with treatment of the underlying malignancy and worsening with relapse supports the notion of DM as a paraneoplastic phenomenon. ${ }^{53}$ In comparison, JDM is noted as a primary disease process and has not been clearly linked with malignancy in children. ${ }^{2,14,53}$ Therefore, screening is not routinely advised unless the history or physical examination provide warning signs for malignancy. It remains unclear why adult and pediatric DMs are histologically and immunologically similar but differ in regard to clinical associations and course.

PM presents in adulthood in the vast majority of cases, and rarely in the pediatric population. The onset of PM is more indolent than JDM/DM, with no heralding skin manifestations, thus making the onset of disease more difficult to pinpoint. Similar to JDM and DM, PM affects the proximal muscles in a symmetric manner, with elevation of muscle enzymes and EMG demonstrating myopathic changes. ${ }^{1,31,57}$ The weakness associated with PM can be more severe than that with DM and affect distal muscles as well. ${ }^{1}$ Unlike JDM, cardiac involvement is more common in patients with PM, occurring in $35 \%$ of cases. ${ }^{1} \mathrm{CK}$ levels in active disease can be elevated to 50 times the normal value, considerably higher than in DM and JDM. ${ }^{31}$ Biopsy in PM demonstrates inflammatory endomysial infiltrates largely composed of $\mathrm{CD} 8+\mathrm{T}$ cells, pointing to a cell-mediated cytotoxic injury involving the myofibers, differentiating it from JDM and $\mathrm{DM}^{1,38} \mathrm{PM}$ is a diagnosis of exclusion after ruling out DM, neuromuscular junction disorders, myotoxic drug exposure, muscle enzyme disease, and muscular dystrophies. ${ }^{31}$ There is a higher incidence of malignancy in adult-onset PM, but not as high as DM, adenocarcinoma being the most commonly associated malignancy. ${ }^{55}$

Similar to JDM, the goals of therapy for DM and PM are decreasing overall disease burden, improving strength, and preventing decline of physical daily function. Corticosteroids and immunosuppressants or modulators remain the mainstays of therapy. ${ }^{58,59}$ If presenting as a paraneoplastic syndrome, treatment of inciting malignancy helps prevent further progression of muscle disease. Patients with PM may present as part of the spectrum of other rheumatologic diseases such as systemic lupus erythematosus and mixed connective tissue disease, thus necessitating longer courses of therapy than those with PM alone. ${ }^{58}$

\section{Immune-Mediated Necrotizing Myopathy}

Considerably rarer and more recently described is the IMNM, also known as necrotizing autoimmune myopathy (NAM). Patients present with severely pronounced proximal muscle weakness similar to JDM, DM, and PM, with a biopsy that demonstrates prominent necrotic changes with minimal or no cellular infiltrate. ${ }^{60}$ This necrosis distinguishes it from the previously described myopathies. Laboratory evaluation reveals elevated muscle enzymes (CK, LDH, ALT, AST), and anti-SRP and anti-hydroxy-3-methylglutaryl-CoA reductase (anti-HMGCR) autoantibodies are highly associated with the disease. ${ }^{60}$ The presence of specific antibodies helps delineate clinical course and treatment. Anti-SRP-positive patients resemble those of JDM and DM without the skin findings. They have more severe muscle involvement, a higher incidence of extramuscular presentations (e.g., interstitial lung disease), and respond better to rituximab. Patients with anti-HMGCR antibodies are typically seen after statin medication use, and respond better to IVIG therapy. Of note, IMNM is different than statin-induced myopathy, which resolves after discontinuation of the offending agent; IMNM continues to persist after ceasing therapy. ${ }^{60,61}$ A seronegative subtype has been described, but there are limited data on the phenomenon and further investigation is required. Though typically presents in the fourth and fifth decades of life, IMNM can present in adolescence, giving a clinical picture similar to that of limb-girdle muscular dystrophy. ${ }^{60}$ The child's weakness may reverse with aggressive early immunosuppression therapy, especially if anti-SRP positive. $^{60,62,63}$ Patients with IMNM have poorer prognosis compared to those with JDM, DM, and PM, demonstrating continued weakness after 2 years of adequate treatment.

\section{Inclusion Body Myositis}

In stark contrast to the previously described myopathies, IBM presents in a distinct fashion that distinguishes it from the other disorders. Classically described as a myopathy presenting after age 50, patients with IBM develop profound weakness and atrophy of the quadriceps and intrinsic flexor muscles of the hands. ${ }^{11}$ One limb is typically more affected than the others. In addition, dysphagia is a common complaint, and may even be the initial complaint given the insidious onset of limb weakness. ${ }^{64}$ Laboratory evaluation demonstrates elevation of the $\mathrm{CK}$, roughly 10 to 15 times the upper limit of normal. ${ }^{11,64}$ Similar to DM and PM, EMG demonstrates myopathic motor units with increased insertional activity, although a few individuals may demonstrate mixed myopathic and 
neuropathic findings. ${ }^{64}$ Muscle biopsy is the definitive test, demonstrating endomysial inflammation, cytoplasmic inclusions, and myofibers with one or more rimmed vacuoles, best seen with Congo red staining. ${ }^{11,64}$ This finding is pathognomonic for the disease.

In contrast to the other inflammatory myopathies, IBM is more treatment resistant to current immunosuppressive agents. In some patients, corticosteroids provide temporary symptomatic relief of symptoms, but resistance usually develops within 3 to 6 years of therapy. ${ }^{64}$ Several studies investigating IVIG as a potential treatment for IBM have demonstrated no benefit, with or without the use of steroids. ${ }^{65,66}$ Similarly, methotrexate, interferon-beta-1a, antithymocyte globulin, oxandrolone, anakinra, and alemtuzumab have all failed to prevent disease progression. ${ }^{64}$ Given the high rates of falls and dysphagia, physical, occupational, and speech therapy are recommended for patients with IBM. Therapy is aimed at building strength and developing safe techniques to accomplish activities of daily living. ${ }^{64}$ Dysphagia is often an underreported symptom, and should be screened for at clinic visits given its high risk of mortality. ${ }^{67}$ Referral to an otolaryngologist may be necessary for patients at risk of aspiration. Cricopharyngeal myotomy, pharyngoesophageal dilatation, or botulinum injection to the upper esophageal sphincter may help maintain oral intake. ${ }^{68}$ For patients who begin to suffer from nutritional deficits and/or weight loss, a feeding tube may be beneficial.

\section{Conclusion}

In summary, the inflammatory myopathies present primarily as muscle weakness, and the configuration of extramuscular manifestations and muscle biopsy help guide diagnosis and treatment of the disease. It is important to recognize that JDM is the most common inflammatory myopathy in children, though PM and IMNM may present in this population as well. Immunosuppression with steroids is the first-line therapy for many of these diseases, though the response to therapy varies depending on the underlying pathology. Early diagnosis and initiation of therapy is key to minimizing the morbidity and mortality associated with these diseases.

\section{Conflict of Interest \\ None declared.}

\section{Acknowledgements}

Neither author has received financial support relevant to this article.

\section{References}

1 Rider LG, Nistala K. The juvenile idiopathic inflammatory myopathies: pathogenesis, clinical and autoantibody phenotypes, and outcomes. J Intern Med 2016;280(01):24-38

2 Feldman BM, Rider LG, Reed AM, Pachman LM. Juvenile dermatomyositis and other idiopathic inflammatory myopathies of childhood. Lancet 2008;371(9631):2201-2212

3 Reed AM, Pachman L, Ober C. Molecular genetic studies of major histocompatibility complex genes in children with juvenile dermatomyositis: increased risk associated with HLA-DQA1 ${ }^{*}$ 0501. Hum Immunol 1991;32(04):235-240
4 Mamyrova G, Rider LG, Ehrlich A, et al. Environmental factors associated with disease flare in juvenile and adult dermatomyositis. Rheumatology (Oxford) 2017;56(08):1342-1347

5 Massa M, Costouros N, Mazzoli F, et al. Self epitopes shared between human skeletal myosin and Streptococcus pyogenes M5 protein are targets of immune responses in active juvenile dermatomyositis. Arthritis Rheum 2002;46(11):3015-3025

6 Bowles NE, Dubowitz V, Sewry CA, Archard LC. Dermatomyositis, polymyositis, and Coxsackie-B-virus infection. Lancet 1987;1 (8540):1004-1007

7 Kwa MC, Silverberg JI, Ardalan K. Inpatient burden of juvenile dermatomyositis among children in the United States. Pediatr Rheumatol Online J 2018;16(01):70

8 Wienke J, Deakin CT, Wedderburn LR, van Wijk F, van RoyenKerkhof A. Systemic and tissue inflammation in juvenile dermatomyositis: from pathogenesis to the quest for monitoring tools. Front Immunol 2018;9:2951

9 Papadopoulou C, McCann LJ. The vasculopathy of juvenile dermatomyositis. Front Pediatr 2018;6:284

10 Lundberg I, Ulfgren A-K, Nyberg P, Andersson U, Klareskog L. Cytokine production in muscle tissue of patients with idiopathic inflammatory myopathies. Arthritis Rheum 1997;40(05):865-874

11 Schmidt J. Current classification and management of inflammatory myopathies. J Neuromuscul Dis 2018;5(02):109-129

12 Harris-Love MO, Shrader JA, Koziol D, et al. Distribution and severity of weakness among patients with polymyositis, dermatomyositis and juvenile dermatomyositis. Rheumatology (Oxford) 2009;48(02):134-139

13 Na S-J, Kim SM, Sunwoo IN, Choi Y-C. Clinical characteristics and outcomes of juvenile and adult dermatomyositis. J Korean Med Sci 2009;24(04):715-721

14 Malleson P. Juvenile dermatomyositis: a review.J R Soc Med 1982; 75(01):33-37

15 Bellutti Enders F, Bader-Meunier B, Baildam E, et al. Consensusbased recommendations for the management of juvenile dermatomyositis. Ann Rheum Dis 2017;76(02):329-340

16 Al-Mayouf SM, AlMutiari N, Muzaffer M, et al. Phenotypic characteristics and outcome of juvenile dermatomyositis in Arab children. Rheumatol Int 2017;37(09):1513-1517

17 Kim S, Kahn P, Robinson AB, et al. Childhood Arthritis and Rheumatology Research Alliance consensus clinical treatment plans for juvenile dermatomyositis with skin predominant disease. Pediatr Rheumatol Online J 2017;15(01):1

18 Sevigny GM, Mathes BM. Periorbital edema as the presenting sign of juvenile dermatomyositis. Pediatr Dermatol 1999;16(01): 43-45

19 Rider LG, Miller FW. Classification and treatment of the juvenile idiopathic inflammatory myopathies. Rheum Dis Clin North Am 1997;23(03):619-655

20 Dicken $\mathrm{CH}$. Periorbital edema: an important physical finding in dermatomyositis. Cutis 1991;48(02):116-117

21 Woo TR, Rasmussen J, Callen JP. Recurrent photosensitive dermatitis preceding juvenile dermatomyositis. Pediatr Dermatol 1985; 2(03):207-212

22 Callen JP. Dermatomyositis. Lancet 2000;355(9197):53-57

23 Christen-Zaech S, Seshadri R, Sundberg J, Paller AS, Pachman LM. Persistent association of nailfold capillaroscopy changes and skin involvement over thirty-six months with duration of untreated disease in patients with juvenile dermatomyositis. Arthritis Rheum 2008;58(02):571-576

24 Smith RL, Sundberg J, Shamiyah E, Dyer A, Pachman LM. Skin involvement in juvenile dermatomyositis is associated with loss of end row nailfold capillary loops. J Rheumatol 2004;31(08): 1644-1649

25 Hoeltzel MF, Oberle EJ, Robinson AB, Agarwal A, Rider LG. The presentation, assessment, pathogenesis, and treatment of calcinosis in juvenile dermatomyositis. Curr Rheumatol Rep 2014;16 (12):467 
26 Lowry CA, Pilkington CA. Juvenile dermatomyositis: extramuscular manifestations and their management. Curr Opin Rheumatol 2009;21(06):575-580

27 Kobayashi I, Yamada M, Takahashi Y, et al. Interstitial lung disease associated with juvenile dermatomyositis: clinical features and efficacy of cyclosporin A. Rheumatology (Oxford) 2003;42(02): 371-374

28 Kobayashi N, Takezaki S, Kobayashi I, et al. Clinical and laboratory features of fatal rapidly progressive interstitial lung disease associated with juvenile dermatomyositis. Rheumatology (Oxford) 2015; 54(05):784-791

29 Volochayev R, Csako G, Wesley R, Rider LG, Miller FW. Laboratory test abnormalities are common in polymyositis and dermatomyositis and differ among clinical and demographic groups. Open Rheumatol J 2012;6:54-63

30 Stringer E, Singh-Grewal D, Feldman BM. Predicting the course of juvenile dermatomyositis: significance of early clinical and laboratory features. Arthritis Rheum 2008;58(11):3585-3592

31 Dalakas MC. Polymyositis, dermatomyositis and inclusion-body myositis. N Engl J Med 1991;325(21):1487-1498

32 Rider LG, Shah M, Mamyrova G, et al; Childhood Myositis Heterogeneity Collaborative Study Group. The myositis autoantibody phenotypes of the juvenile idiopathic inflammatory myopathies. Medicine (Baltimore) 2013;92(04):223-243

33 Zampieri S, Ghirardello A, Iaccarino L, Tarricone E, Gambari PF, Doria A. Anti-Jo-1 antibodies. Autoimmunity 2005;38(01):73-78

34 Ukichi T, Yoshida K, Matsushima S, et al. MRI of skeletal muscles in patients with idiopathic inflammatory myopathies: characteristic findings and diagnostic performance in dermatomyositis. RMD Open 2019;5(01):e000850

35 Ladd PE, Emery KH, Salisbury SR, Laor T, Lovell DJ, Bove KE. Juvenile dermatomyositis: correlation of MRI at presentation with clinical outcome. AJR Am J Roentgenol 2011;197(01):W153-W158

36 Pinto B, Janardana R, Nadig R, et al. Comparison of the 2017 EULAR/ACR criteria with Bohan and Peter criteria for the classification of idiopathic inflammatory myopathies. Clin Rheumatol 2019;38(07):1931-1934

37 Naim MY, Reed AM. Enzyme elevation in patients with juvenile dermatomyositis and steroid myopathy. J Rheumatol 2006;33 (07):1392-1394

38 Vattemi G, Mirabella M, Guglielmi V, et al. Muscle biopsy features of idiopathic inflammatory myopathies and differential diagnosis. Auto Immun Highlights 2014;5(03):77-85

39 Stringer E, Feldman BM. Advances in the treatment of juvenile dermatomyositis. Curr Opin Rheumatol 2006;18(05):503-506

40 Spencer CH, Hanson V, Singsen BH, Bernstein BH, Kornreich HK, King KK. Course of treated juvenile dermatomyositis. J Pediatr 1984;105(03):399-408

41 Fisler RE, Liang MG, Fuhlbrigge RC, Yalcindag A, Sundel RP. Aggressive management of juvenile dermatomyositis results in improved outcome and decreased incidence of calcinosis. J Am Acad Dermatol 2002;47(04):505-511

42 Bowyer SL, Blane CE, Sullivan DB, Cassidy JT. Childhood dermatomyositis: factors predicting functional outcome and development of dystrophic calcification. J Pediatr 1983;103(06):882-888

43 Martin N, Li CK, Wedderburn LR. Juvenile dermatomyositis: new insights and new treatment strategies. Ther Adv Musculoskelet Dis 2012;4(01):41-50

44 Heckmatt J, Hasson N, Saunders C, et al. Cyclosporin in juvenile dermatomyositis. Lancet 1989;1(8646):1063-1066

45 Zeller V, Cohen P, Prieur AM, Guillevin L. Cyclosporin a therapy in refractory juvenile dermatomyositis. Experience and longterm followup of 6 cases. J Rheumatol 1996;23(08):1424-1427

46 Lam CG, Manlhiot C, Pullenayegum EM, Feldman BM. Efficacy of intravenous Ig therapy in juvenile dermatomyositis. Ann Rheum Dis 2011;70(12):2089-2094
47 Oddis CV, Reed AM, Aggarwal R, et al; RIM Study Group. Rituximab in the treatment of refractory adult and juvenile dermatomyositis and adult polymyositis: a randomized, placebo-phase trial. Arthritis Rheum 2013;65(02):314-324

48 Riley P, McCann LJ, Maillard SM, Woo P, Murray KJ, Pilkington CA. Effectiveness of infliximab in the treatment of refractory juvenile dermatomyositis with calcinosis. Rheumatology (Oxford) 2008; 47(06):877-880

49 Dagher R, Desjonquères $\mathrm{M}$, Duquesne $\mathrm{A}$, et al. Mycophenolate mofetil in juvenile dermatomyositis: a case series. Rheumatol Int 2012;32(03):711-716

50 Hassan J, van der Net JJ, van Royen-Kerkhof A. Treatment of refractory juvenile dermatomyositis with tacrolimus. Clin Rheumatol 2008;27(11):1469-1471

51 Batthish M, Feldman BM. Juvenile dermatomyositis. Curr Rheumatol Rep 2011;13(03):216-224

52 Marvi U, Chung L, Fiorentino DF. Clinical presentation and evaluation of dermatomyositis. Indian J Dermatol 2012;57(05):375-381

53 Tansley SL, McHugh NJ, Wedderburn LR. Adult and juvenile dermatomyositis: are the distinct clinical features explained by our current understanding of serological subgroups and pathogenic mechanisms? Arthritis Res Ther 2013;15(02):211

54 Rider LG, Lachenbruch PA, Monroe JB, et al; IMACS Group. Damage extent and predictors in adult and juvenile dermatomyositis and polymyositis as determined with the myositis damage index. Arthritis Rheum 2009;60(11):3425-3435

55 Hill CL, Zhang Y, Sigurgeirsson B, et al. Frequency of specific cancer types in dermatomyositis and polymyositis: a population-based study. Lancet 2001;357(9250):96-100

56 Morris P, Dare J. Juvenile dermatomyositis as a paraneoplastic phenomenon: an update. J Pediatr Hematol Oncol 2010;32(03): 189-191

57 Mastaglia FL, Phillips BA. Idiopathic inflammatory myopathies: epidemiology, classification, and diagnostic criteria. Rheum Dis Clin North Am 2002;28(04):723-741

58 Hunter K, Lyon MG. Evaluation and management of polymyositis. Indian J Dermatol 2012;57(05):371-374

59 Sasaki H, Kohsaka H. Current diagnosis and treatment of polymyositis and dermatomyositis. Mod Rheumatol 2018;28(06):913-921

60 Pinal-Fernandez I, Casal-Dominguez M, Mammen AL. Immune-mediated necrotizing myopathy. Curr Rheumatol Rep 2018;20(04):21

61 Mammen AL, Tiniakou E. Intravenous immune globulin for statintriggered autoimmune myopathy. N Engl J Med 2015;373(17): $1680-1682$

62 Valiyil R, Casciola-Rosen L, Hong G, Mammen A, Christopher-Stine L. Rituximab therapy for myopathy associated with anti-signal recognition particle antibodies: a case series. Arthritis Care Res (Hoboken) 2010;62(09):1328-1334

63 Suzuki S, Ohta M, Shimizu Y, Hayashi YK, Nishino I. Anti-signal recognition particle myopathy in the first decade of life. Pediatr Neurol 2011;45(02):114-116

64 Dimachkie MM, Barohn RJ. Inclusion body myositis. Neurol Clin 2014;32(03):629-646, vii

65 Dalakas MC, Koffman B, Fujii M, Spector S, Sivakumar K, Cupler E. A controlled study of intravenous immunoglobulin combined with prednisone in the treatment of IBM. Neurology 2001;56 (03):323-327

66 Dalakas MC, Sonies B, Dambrosia J, Sekul E, Cupler E, Sivakumar K. Treatment of inclusion-body myositis with IVIg: a double-blind, placebo-controlled study. Neurology 1997;48(03):712-716

67 Cox FM, Verschuuren JJ, Verbist BM, Niks EH, Wintzen AR, Badrising UA. Detecting dysphagia in inclusion body myositis. J Neurol 2009;256(12):2009-2013

$68 \mathrm{Oh} \mathrm{TH}$, Brumfield KA, Hoskin TL, Kasperbauer JL, Basford JR. Dysphagia in inclusion body myositis: clinical features, management, and clinical outcome. Am J Phys Med Rehabil 2008;87(11):883-889 
